\title{
Approximation et précision : deux facettes d'une même réalité
}

\author{
Asnes, Maria \\ Collège Beit Berl \\ maria.asnes@gmail.com
}

\section{Introduction}

L'approximation et la précision semblent être à deux pôles opposés: en effet, l'approximation est souvent considérée comme l'imprécision étant donné qu'elle fournit une représentation inexacte d'une valeur connue ou inconnue. Pourtant, la langue nous confronte avec des cas où le même terme peut avoir un effet de sens tantôt approximatif tantôt précis. S'agit-il alors de deux emplois complètement distincts ou de deux effets de sens issus du même noyau sémantique?

Cette étude prend comme objet l'adverbe plutôt qui manifeste des emplois approximatifs ainsi que précis. A travers l'étude de ce terme on montrera que l'approximation et la précision ne sont pas toujours des termes opposés, mais qu'ils relèvent de deux facettes d'une même réalité de base.

Précisons que l'approximation et la précision ne sont que des effets de sens dérivés. L'intérêt de notre recherche ne consiste pas à présenter le noyau sémantique de plutôt mais de montrer à travers ce terme que l'approximation et la précision ne sont pas toujours des notions complémentaires.

La section 1 présente les valeurs sémantiques de l'adverbe plutôt. Les emplois relevés sont classés dans la section 2 en termes d'approximation et précision. L'existence de ces deux types d'emplois présente un paradoxe apparent, qui sera résolu dans la section 3.

\section{Valeurs sémantiques de plutôt}

\subsection{Parcours diachronique}

La diversité des emplois de plutôt est un résultat du processus de grammaticalisation que ce terme a subi.

A l'origine il s'agit de l'emploi temporel (plus tôt ou plutôt) qui suppose une comparaison objective entre deux éléments:

(1) Il est arrivé plus tôt que prévu.

(2) Pas plutôt Jaume parti, du bout de la ruelle j'ai vu arriver une forme noire, haute, mince, si mince que, d'abord, j'ai cru que je rêvais. (GIONO, Colline, 1929, p.100).

Selon Mokni (2008), le parcours diachronique de plutôt manifeste de la grammaticalisation et montre une évolution vers plus de subjectivité : Temps $\rightarrow$ Choix Préférentiel $\rightarrow$ Opposition

Mokni constate que plutôt peut exprimer, entre autres, la préférence et la rectification énonciative. Tandis que l'emploi temporel suppose une évaluation objective des deux éléments de la comparaison, l'emploi préférentiel est lié à une évaluation faite par le locuteur. La rectification, à son tour, consiste à rejeter une assertion précédente et à exprimer sa préférence pour une autre. Les emplois de choix préférentiel et de rectification (opposition) sont donc des emplois subjectifs, étant donné que le locuteur exprime une préférence dans une comparaison ou dans une énonciation. 


\subsection{Valeurs sémantiques attestées}

La distribution syntaxique de l'adverbe plutôt relève d'une grande diversité. Chacun de ses emplois syntaxiques est accompagné d'un effet de sens particulier.

Les emplois discutés dans cette section sont attestés en français moderne.

Selon le Trésor de la Langue Française, plutôt apparaît dans les contextes suivants:

\section{A. Marque de préférence}

\section{A1. Possibilité préférée}

(3) M. le Proviseur reçoit demain toute la journée. Venez plutôt le matin. Venez plutôt vers huit heures (MALÈGUE, Augustin, t.1, 1933, p.152)

Lorsqu'il y a le choix entre deux possibilités, plutôt indique la possibilité préférée. La possibilité la moins préférée peut être soit implicite (4) soit explicite (5):

(4) J'ai dit à Robert que je le trouvais bien imprudent d'encourager ainsi un raté qu'il vaudrait mieux pousser à faire n'importe quoi plutôt que de la peinture... (GIDE, École femmes, 1929, p.1269)

A2. Rectification (synonyme de plus exactement)

(5) J'y ai rencontré une jeune fille, une jeune femme, plutôt (COCTEAU, Parents, 1938, I, 4, p.206)

Dans ce type d'emploi, la valeur rectifiée (une jeune fille) est normalement exprimée explicitement.

\section{B. Modification du degré}

B1. Downtoning (synonyme d'assez, pas mal)

(6) Nos rapports étaient plutôt tendus (MALRAUX, Conquér., 1928, p.20).

Le terme de base par rapport auquel se fait la comparaison est absent.

B2. Intensification par litote (synonyme de très, beaucoup)

(7) Faut-il tout prendre? Prenez, prenez. Mais il tient un cercle? C'est plutôt mal vu. Plutôt, oui (RENARD, Journal, 1908, p.1149).

\subsection{Synthèse et quelques valeurs supplémentaires}

La revue des valeurs attestées de plutôt révèle que cet adverbe a deux valeurs principales: celle de modification de degré et celle de rectification. Pourtant, on s'aperçoit que les dictionnaires n'attestent pas tous les emplois possibles de cet adverbe. Dans ce qui suit, on établit une synthèse des emplois attestés et non-attesté de plutôt avant d'aborder la discussion sur le rapport avec les notions d'approximation et de précision (section 3).

\subsubsection{Modification de degré}

Lorsque plutôt précède un terme scalaire, il modifie le degré d'une propriété:

(8) Elle est plutôt belle.

(9) Cette robe est plutôt magnifique!

Belle dans (8) et magnifique dans (9) dénotent des propriétés scalaires. Lorsque ces termes sont précédés de plutôt, le degré posé d'une propriété (plutôt $\mathrm{P}$ ) est mis en rapport avec son degré standard (P). Ainsi, dans (8), la propriété d'être plutôt belle est mise en rapport avec la propriété d'être belle. 
La position du degré posé par rapport au degré standard est pourtant différente dans (8) et dans (9). Dans (8), le degré posé (plutôt belle) semble être inférieur par rapport au degré standard (belle). Ainsi, plutôt est paraphrasable par assez et fonctionne donc comme un downtoner :

(8') Elle est assez belle.

L'énoncé (8) est normalement prononcé dans les circonstances où la personne dont on parle n'est pas jugée comme correspondant à l'étalon de la beauté. Cette personne n'est pas vraiment belle, mais elle est dite avoir une sorte de beauté, une beauté particulière.

Par contre, dans (9), plutôt fonctionne comme un intensifieur. Cela est sans doute dû à l'effet produit par le degré superlatif de l'adjectif magnifique qui est dans sa portée. La paraphrase avec assez n'est pas possible ici. On paraphrasera cet énoncé plutôt par absolument :

(9') Cette robe est absolument magnifique.

Malgré la paraphrase, plutôt contrairement à absolument, indique qu'il s'agit d'une opinion subjective du locuteur.

Contrairement aux exemples (8) et (9) où plutôt précède un terme scalaire à échelle ouverte, il peut aussi précéder un terme scalaire à échelle fermée:

(10) Votre réponse est plutôt fausse.

Dans ce cas, la réponse est considérée comme fausse, mais peut être pas "totalement fausse". Le locuteur utilise plutôt pour atténuer la force de l'assertion en la présentant comme une vérité subjective. Remarquons que, dans les sondages où les répondeurs doivent asserter la vérité des propositions, il y a souvent 4 possibilités de réponse: totalement vrai, plutôt vrai, plutôt faux, totalement faux. On constate donc que sur le continuum de degrés, plutôt occupe une position inférieure par rapport à totalement.

Pour en conclure, plutôt devant un terme scalaire modifie le degré de la propriété posée. Le type de modification (intensification ou downtoning) dépend du type de propriété :

- avec des propriétés à échelle ouverte au degré neutre plutôt fonctionne comme un downtoner

- avec les propriétés à échelle ouverte au degré superlatif il opère l'intensification.

- avec les propriétés à échelle fermée, il fonctionne comme un downtoner en ce sens qu'il attenue la force de l'assertion.

Dans tous les cas il s'agit d'assertions subjectives du locuteur et donc la vérité de la proposition est relative à l'univers de croyances du locuteur.

\subsubsection{Rectification}

A part les emplois où plutôt modifie le degré d'une propriété scalaire, cet adverbe peut dénoter également une rectification du contenu précédent.

Dans cet emploi, plutôt est normalement précédé des conjonctions ou ou mais.

(11) Je suis à Paris, ou plutôt à Saint-Germain, depuis trois jours.

(12) Je ne suis pas inquiet, mais plutôt vigilant

Dans les exemples (11) et (12) plutôt $\mathrm{P}$ rectifie l'information précédente $(\mathrm{P})$ :

Sant-Germain est une rectification par rapport à Paris

Vigilant est une rectification par rapport à inquiet. 
Schapira (2010) dans sont étude sur plus précisément/plus exactement propose de distinguer deux types de ce qu'on appelle ici rectification: restriction et rectification (proprement dite). On va se baser sur cette dichotomie pour préciser davantage les valeurs sémantiques de plutôt.

\section{a. Restriction}

Avant de discuter de l'emploi restrictif à propos de l'adverbe plutôt, regardons en quoi consiste cet emploi sur l'exemple des adverbes plus précisément et plus exactement.

Selon Schapira (2010), dans cet emploi, les expressions adverbiales en question opèrent une restriction du champ de référence du contenu précédent. Le contenu introduit par plus précisément/plus exactement est inclus à l'intérieur du contenu précédent. Le contenu précédent n'est pas faux, mais le contenu posé est dit être plus précis.

(13) Les jeux olympiques d'hiver auront lieu cette année en Autriche et plus précisément/plus exactement à Innsbruck.

(14) Anne veut acheter des meubles et plus précisément/plus exactement une table et des chaises.

(Schapira 2010:18).

Innsbruck et Autriche (14) sont des termes perçus comme assez proches. La relation entre ces termes est celle de sous-ensemble (Innsbruck est une ville en Autriche):

(13') Innsbruck $\subset$ Autriche

De même, table et chaises représentent un sous-ensemble des meubles.

(14') table $\subset$ meubles

chaises $\subset$ meubles

Plutôt peut avoir la même valeur de restriction que plus précisément et plus exactement:

(15) Vendredi dernier nous sommes parties en expédition à Paris, ou plutôt, à la porte de Versailles.

La porte de Versaille est un endroit à l'intérieur de Paris:

$\left(15^{\prime}\right)$ porte de Versailles $\subset$ Paris

\section{b. Extension}

Cet emploi où plutôt opère une extension par rapport à l'information précédente n'est pas attesté par les dictionnaires.

(16) Le français des dictionnaires est le français de Paris ou plutôt de la région de l'Ile-de-France, issu du dialecte d'oill.

Cet emploi ressemble l'emploi restrictif, en ce que les termes mis en relation sont perçus comme proches ou appartenant au même champ de référence. Pourtant, la relation entre l'information précédente (P1) et l'information nouvelle (P2) est inverse. A savoir, bien que l'information nouvelle précise l'information précédente, elle dénote un champ plus large que l'information précédente:

(16') Paris $\subset$ Ile de France

\section{c. Rectification proprement dite}

Selon Schapira (2010), cet emploi n'est propre qu'à plus exactement Dans cet emploi, plus exactement ne signifie pas la restriction, mais la rectification, à savoir l'information introduite par plus exactement ne limite pas l'information précédente (comme dans l'emploi restrictif), mais plutôt l'annule.

(17) Pierre a perdu son parapluie ou, plus exactement, on le lui a volé. (Schapira 2010:19). 
L'information qui suit plus exactement annule l'assertion précédente en la posant comme fausse. Cette nouvelle information est considérée comme conforme à la réalité ou vraie.

(17') $[[$ Pierre a perdu son parapluie $]]=$ faux, $[[$ on le lui a volé $]]=$ vrai

Contrairement à la restriction et l'extension, il n'y a pas de relation d'inclusion entre les deux termes, mais ils sont plutôt membres du même paradigme à un même niveau linéaire.

(17") Les clés ne sont pas là

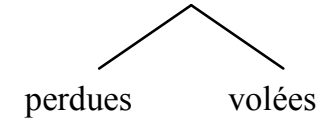

Plutôt tout comme plus exactement peut avoir la valeur de rectification proprement dite:

(18) Pierre a perdu son parapluie ou, plutôt, on le lui a volé.

$\left(18^{\prime}\right)$ Pierre a perdu son parapluie $\neq$ on lui a volé le parapluie

Remarquons que tout comme plus précisément et plus exactement présentent le degré comparatif des adverbes précisément et exactement respectivement, plutôt présente aussi un degré comparatif bien qu'il ne soit plus décomposable en plus et tôt.

Outre des emplois restrictif et rectificatif synonymiques de plus précisément/plus exactement, plutôt peut avoir des emplois supplémentaires qui ne sont pas attestés par les dictionnaires (voir ci-dessous).

\section{d. Probabilité, proximité à la vérité}

Un emploi dans lequel plutôt met l'information posée en relation avec la vérité est illustré par les exemples suivants:

(19) Géographiquement, ça se passerait plutôt à Paris, ou en proche banlieue.

(20) Et en tous cas pour mes prochaines vacances j'irai plutôt à New York.

Dans les exemples (19) et (20), plutôt est paraphrasable par probablement. Autrement dit, l'information posée est présentée comme proche de la vérité mais pas totalement certaine. Plutôt ici fonctionne à nouveau comme un opérateur subjectif qui relativise la vérité de la proposition à l'univers de croyances du locuteur.

Cet emploi pourrait être rangé dans la catégorie des emplois rectificatifs puisqu'il relève d'une relation avec la vérité de la proposition. Pourtant, contrairement aux emplois rectificatifs discutés ci-dessus, dans cet emploi il n'y a pas de comparaison entre l'information précédente et l'information nouvelle. Le deuxième terme de comparaison est absent ou sous-entendu. Ce qui se passe ici c'est que l'information posée est dite n'être vraie que probablement.

\section{e. Généralisation, vérité dans la majorité des cas}

(21) En Norvège, les habitants aisés habitent plutôt à l'extérieur de la ville, et peuvent payer pour entrer en voiture dans le centre ville.

(22) Les références présentées viennent plutôt de scientifiques universitaires en sciences sociales aux Etats-Unis et en Grande-Bretagne, mais aussi de chercheurs gouvernementaux et de psychologues.

Dans cet emploi l'information introduite par plutôt est présentée comme vraie dans la majorité des cas. Contrairement à l'emploi de probabilité (d), il s'agit ici d'une information objective, mais qui n'est vraie que dans la majorité des cas.

La paraphrase possible de (21) et (22) serait (21') et (22') où la valeur de plutôt est assumée par le quantifieur nominal la plupart de:

(21') En Norvège, la plupart des habitants aisés habitent à l'extérieur de la ville... 
(22') La plupart des références présentées viennent de scientifiques universitaires en sciences sociales aux Etats-Unis et en Grande-Bretagne...

\section{f. Possibilité préférée}

Plutôt peut également désigner l'option préférée. L'option moins préférée peut être soit exprimée explicitement, soit sous-entendue. Dans cet emploi plutôt opère comme un terme comparatif avec un effet modal de volonté:

(1) Venez plutôt le matin = il est préférable que vous veniez le matin et non dans l'après midi ou le soir.

(23) Allons à la campagne plutôt que de rester à Paris

Dans (1), la possibilité posée est indiquée comme la plus préférée. Les autres possibilités sont laissées sous-entendues.

Dans (23) la conjonction plutôt que établit une comparaison entre deux possibilités, l'une desquelles est marquées comme préférée.

On pourrait ranger cet emploi dans la catégorie de rectification, puisque la possibilité préférée est jugée comme vraie dans le monde possible qui est celui de la volonté du locuteur.

\section{Approximation et précision: deux effets de sens de plutôt}

L'approximation et la précision sont deux notions qui constituent un domaine de recherche important non seulement en langue naturelle mais aussi dans les sciences exactes. Tandis que l'approximation fait référence à une représentation inexacte d'une valeur connue ou inconnue, la précision dénote la proximité de deux valeurs l'une par rapport à l'autre. Il est intéressant de remarquer que, dans les deux cas, celui d'approximation et de précision, il s'agit d'une proximité entre deux valeurs. Il apparaît donc qu'il y a deux façons de rendre compte de la proximité. L'approximation focalise le fait qu'une valeur est proche mais inexacte. Par contre, la précision asserte qu'une valeur se rapproche de la valeur exacte et devient presque indiscernable de celle-ci.

L'examen des valeurs sémantiques de plutôt nous montre déjà que ce terme est étroitement lié au domaine d'approximation et de précision. Etant un terme comparatif de base, plutôt met en relations deux valeurs. On constate que, dans différents contextes, la relation entre ces valeurs relève tantôt de l'approximation tantôt de la précision. Cela présente un paradoxe: comment le même terme peut avoir deux effets de sens tellement différents et même opposés?

Dans cette section on classera et analysera les valeurs sémantiques présentées ci-dessus en termes d'approximation et de précision. Ce classement nous servira de base pour la résolution de ce paradoxe apparent dans la section 3 .

\subsection{Effets de sens d'approximation}

\section{a. Modification de degré}

Lorsque plutôt fonctionne comme un modifieur de degré, l'expression modifiée par plutôt a la valeur approximative, étant donné qu'elle présente un degré subjectif de la propriété posée.

Dans (8), plutôt belle signifie qu'il s'agit d'un degré inférieur par rapport au degré standard. Dans (9), plutôt magnifique désigne le degré supérieur par rapport au degré standard.

Dans les deux cas, plutôt désigne un degré qui s'éloigne par rapport au degré standard d'être belle.

Si on veut généraliser à partir des exemples (8) et (9), on dira que le fait d'avoir la propriété d'être plutôt $P$ pour un objet est vrai si et seulement si le degré de cette propriété (dénoté par plutôt $P$ ) est inférieur (8) ou supérieur (9) au degré de la propriété $P$ où le degré de la propriété $P$ est considéré comme le degré standard. 


\section{b. Probabilité, proximité à la vérité}

La valeur de probabilité, bien que rangée parmi les emplois de rectification dans ce qui précède, relève en fait de l'approximation étant donné qu'on se rapproche de la vérité, mais il y a quand même un certain degré d'incertitude (exemples 21, 22).

La proposition modifiée par plutôt est vraie dans un monde réel si et seulement si il existe un monde possible qui est proche du monde réel et que, dans ce monde possible, la proposition non-modifiée par plutôt est vraie.

La vérité est présentée comme vérité dans un monde possible qui est pourtant très proche du monde réel selon l'univers de croyance du locuteur.

\section{c. Possibilité préférée}

Lorsque la possibilité préférée n'est pas explicitement mise en rapport avec une autre possibilité, nous avons un effet d'approximation. Ainsi, dans (1), plutôt le matin signifie de façon imprécise l'intervalle de temps pendant lequel on pourrait venir.

La proposition modifiée par plutôt est vraie dans un monde réel si et seulement si il existe un monde possible dans lequel la proposition non-modifiée par plutôt est vraie. La différence de cet emploi par rapport à l'emploi de probabilité est la distance entre le monde possible et le monde réel.

\section{d. Généralisation, vérité dans la majorité des cas}

La valeur de généralisation relève de l'approximation puisqu'il s'agit à nouveau d'une évaluation, cette fois-ci en termes de majorité. La quantité exacte n'est pas connue mais il y a une estimation approximative de la quantité (exemples 21,22)

Pour tout individu qui a une certaine propriété $P$ (e.g. d'être résident aisé de Norvège), le fait d'avoir aussi la propriété de plutôt $Q$ (e.g. d'habiter à l'extérieur de la ville), implique que la cardinalité d'individus ayant la propriété $P$ et la propriété $Q$ est plus grande que la cardinalité des individus ayant la propriété $\mathrm{P}$ et n'ayant pas de propriété Q.

\subsection{Effets de sens de précision}

\section{a. Restriction}

La restriction consiste en précision du champ dénotationnel du concept. Dans cet emploi plutôt est paraphrasable par plus précisément ou plus exactement.

L'expression précédée de plutôt est plus précise que son antécédent si et seulement si la dénotation de cette expression est un sous-ensemble (strict) de son antécédent.

\section{b. Extension}

Extension est le même procédé que la restriction mais qui va dans un sens inverse. La valeur posée est précisée non pas par un terme avec une extension plus étroite mais, au contraire, par un terme ayant une extension plus grande. Il n'en reste pas moins qu'il s'agit d'un procédé de précision, puisque l'information nouvelle est posée comme plus précise que la première information.

L'expression précédée de plutôt est plus précise que son antécédent si et seulement la dénotation de son antécédent est incluse dans sa dénotation et si, pour toute propriété qui est incluse dans la propriété exprimée par une expression modifiée par plutôt, la proposition sera vraie.

\section{c. Rectification proprement dite}

Rectification proprement dite est étroitement liée à la notion de vérité. Il s'agit d'une correction d'une erreur. 
La rectification diffère de la précision par restriction ou par extension en ce qu'il n'y a pas de relation de sous-ensemble entre la valeur introduite par plutôt et la valeur antécédente.

La proposition modifiée par plutôt est une rectification d'une autre proposition si est seulement si la deuxième proposition est fausse et la proposition modifiée par plutôt est vraie et et sil n'y pas de relation de sous-ensemble entre les deux propositions.

\section{d. Possibilité préférée (comparaison entre deux termes)}

Lorsque plutôt que établit une comparaison entre deux possibilités explicites, l'une des possibilités est marquée comme étant la plus préférée. Il s'agit d'une sorte de rectification, mais dans un monde possible de préférences.

On vient de constater que plutôt manifeste à travers ses différentes valeurs tantôt la valeur d'approximation tantôt celle de précision. Ceci constitue en apparence un paradoxe qu'on essaiera de résoudre dans ce qui suit.

\section{Paradoxe résolu}

\subsection{Approximation et précision: deux facettes d'une même réalité}

Adler et Asnes (2013, à par.) ont tenté de définir les notions d'approximation et de précision. On résume ci-dessous les concepts-clés.

\subsubsection{Précision (et Exactitude)}

La notion de précision doit être étudiée en corrélation avec celle d'exactitude. Dans l'usage courant, ces deux notions sont considérées comme synonymiques. Cependant, en sciences exactes, on les utilise pour référer à deux réalités différentes.

Ainsi, la précision est une mesure de la fidélité/fiabilité de l'expérience, ou la possibilité pour l'expérience d'être reproductible. L'exactitude, par contre, est liée à la conformité des résultats à une valeur vraie.

Schapira (2010) discute de la différence linguistique entre la précision et l'exactitude à propos des connecteurs plus précisément/plus exactement. Alors que plus précisément peut être utilisé à la fois pour préciser et rectifier l'information antérieure, plus exactement ne peut que la rectifier. Préciser, dans le sens de Schapira, serait de fournir l'information qui est jugée comme proche de l'information antérieure mais ayant le champ de référence plus limité. Nous avons montré dans ce qui précède, que l'information nouvelle peut être jugée plus précise également si elle élargit le champ référentiel de l'antécédent. Rectifier, par contre, serait annuler l'information antérieure en fournissant l'information nouvelle est qui jugée comme vraie.

\subsubsection{Approximation}

L'approximation ne peut pas être tout simplement réduite à être le contraire de la précision. Etant donné qu'on distingue la précision et l'exactitude, la question est donc de savoir si l'approximation relève de l'imprécision ou de l'inexactitude.

Selon Adler et Asnes (à par.), l'approximation se trouve à l'intersection de l'imprécision et de l'exactitude relative soit par rapport au monde réel soit par rapport à l'univers des croyances du locuteur. Ce qui peut paraître étonnant selon cette définition, c'est le rapport entre l'approximation et l'exactitude. L'approximation présuppose l'exactitude (étant donné que l'exactitude peut caractériser un intervalle de valeurs) toute en étant une imprécision.

(24) Marie a près de 55 ans 
a. imprécis, exact si l'âge de Marie est réellement près de 55 ans

b. imprécis, inexact si l'âge de Maris n'est pas près de 55 ans

(25) Marie a $\mathbf{5 0}$ ans

a. précis, exact si l'âge de Maris est 50 ans

b. précis, inexact, si l'âge de Marie n'est pas 50 ans

c. imprécis, exact si 50 ans signifie "à peu près 50 ans" et si l'âge de Maris est 50 ans

d. imprécis, inexact si 50 ans signifie "à peu près 50 ans" et si l'âge de Maris n'est pas à peu près 50 ans

L'approximation relève tout d'abord de l'éloignement de la valeur précise et donc de l'imprécision. Pourtant, on ne peut parler d'une approximation que si la valeur dont elle s'éloigne est jugée comme vraie. Quand on dit d'une proposition que ceci est une approximation on suppose que cette approximation est proche de la vérité. Il n'est pas clair ce qui signifierait d'être approximatif par rapport à la valeur fausse. La vérité peut être une vérité dans le monde réel ou dans un monde de croyance du locuteur. S'il s'agit de la vérité dans le monde réel, l'approximation est exacte. S'il s'agit de la vérité dans l'univers de croyance du locuteur l'approximation peut être inexacte dans le monde réel.

Cependant, si le locuteur consciemment fournit une valeur fausse, il s'agirait tout simplement d'une imprécision et d'une fausseté.

On pourrait encore reformuler ce contraste en disant que dans le domaine de la vérité, on peut avoir la vérité exprimée de façon précise ou imprécise (approximative). Dans le domaine du faux, toutes les valeurs sont fausses, on peut donc parler d'une façon précise ou imprécise, mais l'approximation dans le sens du rapprochement de la vérité n'existe pas.

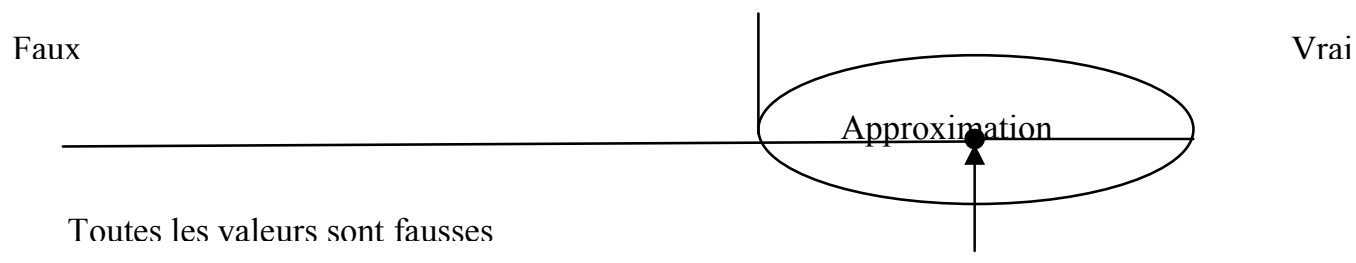

Valeur vraie

\subsection{Résolution du paradoxe}

Plutôt est un terme essentiellement comparatif qui établit la comparaison par rapport à la valeur standard.

Il y a deux facteurs qui déterminent la valeur approximative ou précise de l'assertion:

1. Comparaison explicite entre 2 valeurs ou comparaison implicite entre la valeur assertée et un standard de comparaison implicite

\begin{tabular}{|l|l|}
\hline Comparaison explicite entre 2 termes & Comparaison implicite \\
\hline Restriction & Modification de degré \\
\hline Extension & Probabilité \\
\hline Rectification proprement dite & Généralisation \\
\hline Possibilité préférée (2 termes) & Possibilité préférée (1 terme) \\
\hline
\end{tabular}


2. La relation avec la valeur de vérité

a. Dans le cas d'une comparaison explicite, la valeur introduite par plutôt est considérée comme étant plus proche de la valeur de vérité que l'autre valeur, d'où l'effet de précision.

b. Dans le cas d'une comparaison implicite, la valeur introduite par plutôt est considérée comme étant éloignée du standard de comparaison où de la valeur vraie, d'où l'effet d'approximation. Pour les propriétés scalaires, l'approximation consiste en une spécification d'un degré qui est soit inférieur ou supérieur par rapport au standard de comparaison. Pour les propriétés non-scalaires, la valeur est considérée être proche mais toutefois distinctes de la valeur vraie.

En fait, avec les propriétés non-scalaires, il s'agit plutôt de l'exactitude que de la précision. On a montré antérieurement que l'exactitude et l'approximation ne se contredisent pas: un terme peut être exact (vrai) mais approximatif (imprécis).

Le noyau sémantique de plutôt est donc d'établir la comparaison avec la valeur standard qui est considérée comme vraie. Cette proximité de la vérité peut être interprétée tantôt comme approximation tantôt comme précision. Si la comparaison se fait avec la valeur vraie (exact), le fait même d'être éloignée de cette valeur favorise l'interprétation approximative.

Par contre, si la comparaison se fait avec une autre propriété, qui est encore plus éloigné de la valeur de vérité, on obtient l'effet de précision.

Eloignement de la valeur standard: Plutôt $\mathrm{P} \leftarrow \mathrm{P}$ [Vérité] - approximation

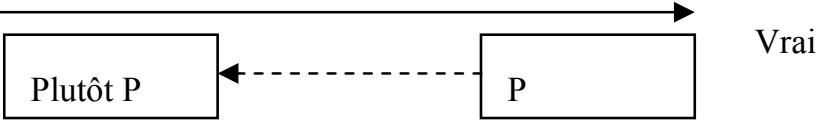

Rapprochement de la vérité: $\mathrm{P} \rightarrow$ Plutôt $\mathrm{P}$ [Vérité] - précision

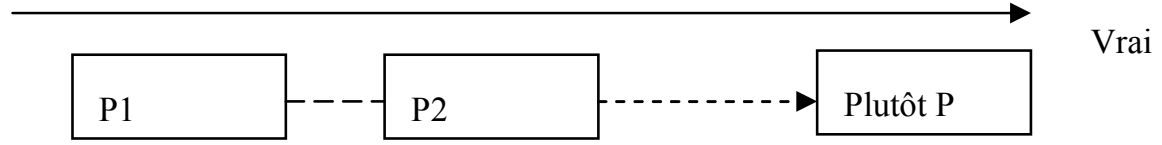

\section{Références bibliographiques}

\section{Dictionnaires}

Trésor de la Langue Française informatisé

\section{Ouvrages linguistiques}

Adler, S., Asnes, M. (2008). Approximation par arrondissement: le cas de quelques quantifieurs prépositionnels. In Durand J., Habert B., Laks B. (éds), Congrès Mondial de Linguistique Française - CMLF 08, Paris - Institut de Linguistique Française.

Adler, S., Asnes, M. (2010a). Autour de la précision. L'Information Grammaticale, 125, 36-43.

Adler, S. Asnes, M. (2010b). Prépositions au service de la scalarité. In Hadermann, P., Inkova, O., Pierrard, M. et Van Raemdonck, D. (éds), Approches de la Scalarité, Genève: Droz, 223-247.

Adler, S., Asnes, M. (2012). A la recherche des quantifieurs perdus. In Schnedecker, C. et Armbrecht C. (éds), $L a$ quantification et ses domaines, Paris: Honoré Champion, 569-582. 
Adler S., Asnes, M. (à par). Quantification approximative et quantification floue: essai de précision. In Bat-Zeev H., Adler S \& Asnes M. (éds), Précis et imprécis: Etude sur l'approximation et la précision, Paris: Champion, $25-42$.

Ariel, M. (2003). Does most mean 'more than half'? BLS, 29, 17-30.

Ariel, M. (2004). Most. Language, 8 (4), 658-706.

Ariel, M. (2006). A 'just that' lexical meaning for most. In von Heusinger K., Turner K. (eds), Where semantics meets pragmatics, Current Research in Semantics / Pragmatics interface, Issy les Moulineaux : Elsevier, 49-94.

Biber, D. et al. (1999). Longman Grammar of Written and Spoken English. London: Longman.

Greenberg, Y., Ronen M., (forthcoming). Three approximators which are almost/more or less/be-gadol the same. Proceedings of IATL 28.

Greenberg, Y., Khrizman, K. (2012). 'Bixlal- a general strengthening operator'. In Proceedings of IATL 27,139-162.

Jayez, J. (1987). Sémantique et approximation : le cas de presque et à peine. Lingvisticae Investigationes, 11.1, 157196.

Jayez J., L. Tovena (2008). Presque and almost: how argumentation derives from comparative meaning. In Bonami

O. \& Cabredo Hofherr P. (eds), Empirical Issues in Syntax and Semantics, 7, 1-25 (http://www.cssp.cnrs.fr/eiss6).

Kennedy, Ch. (2011). Vagueness and Comparison. In P. Egre, N. Klinedinst, Vagueness and Language Use. Palgrave MacMillan.

Kennedy, Ch. (2012). Ambiguity and Vagueness: an Overview. In Maienborn C., von Heusinger K. and 1 Portner P. (eds), The Handbook of Semantics, Berlin: Mouton de Gruyter, 507-552.

Kennedy, Ch. (2013). A scalar semantics for scalar readings of number words. In Caponigro I. \& Cecchetto C. (eds), From grammar to meaning: The spontaneous logicality of language, Cambridge : Cambridge University Press, $172-200$.

Kennedy, Ch., and McNally L. (2005). Scale Structure, Degree Modification, and the Semantics of Gradable Predicates. Language, 81(2), 345-381.

Krifka, M. (2002). Be brief and vague! And how bidirectional optimality theory allows for verbosity and precision. In Restle D. \& Zaefferer D. (eds), Sounds and Systems. Studies in Structure and Change. A Festschrift for Theo Vennemann, Berlin: Mouton de Gruyter, 439-458.

Krifka, M. (2007). Approximate interpretation of number words: A case for strategic communication. In Zwarts J., Bouma G., Krämer I. (eds), Cognitive Foundations of Interpretation, Amsterdam: Mouton de Gruyter, 111126.

Krifka, M. (2012). Approximate and Precise Use of Language, a talk given in the conference Vague Language Vague Law?, Albert-Ludwigs-Universität Freiburg, January 20 - 21, 2012. http://amor.cms.huberlin.de/ h2816i3x/Talks/VagheitFreiburg.pdf

Mihatsch, W. (2009). L'approximation entre sens et signification : un tour d'horizon. In Verbeken, D. (éd.), Entre sens et signification, Paris : L'Harmattan, 125-144.

Mihatsch, W. (2010a). Les approximateurs quantitatifs entre scalarité et non-scalarité. Langue française, 165, 125153.

Mihatsch, W. (2010b). The diachrony of rounders and adaptors : approximation and unidirectional change. In Kaltenbock, G., Mihatsch, W. \& S. Schneider (eds), New Approaches to Hedging, Bingley : Emerald, 93-122.

Mokni, M. (2008). La grammaticalisation de l'adverbe plutôt et l'évolution du système grammatical. Linx, 59, 171184.

Romero, C. (2005). L'adjectif en français et à travers les langues, In François J. (éd.), L'adjectif en français et à travers les langues, Caen : Presses universitaires de Caen, 449-462.

Schapira, C. (2010). Précision ou, plus exactement, focalisation discursive, L'Information Grammaticale, 125, 17-21. 
Weidman-Sassoon, G., Zevakhina, N. (à par.). Granularity shifting: Experimental evidence from numerals vs. adjectives. Proceedings of the IATL 28. MIT Working Papers in Linguistics.

Weidman-Sassoon, G. (2013). Vagueness, Gradability, and Typicality - The Interpretation of Adjectives and Nouns.

In Turner K. P. and von Heusinger K. (eds), Current Research in the Semantics Pragmatic Interface series (CRiSPI) 27, Leiden : Brill.

Ziv, Y. (2013). Hebrew day/dey as a modifying approximator and its illocutionary force, presentation orale au colloque international. "Approximation and Precision III", Université de Tel Aviv \& Université Bar Ilan, 1617 juin 2013. 